\title{
A NOTE ON BERNOULLI AND EULER NUMBERS
}

OF ORDER $\pm p$

L. CARLITZ

1. Introduction. The Bernoulli numbers of order $k$ may be defined by $[6$, p. 143]

$$
\left(\frac{x}{e^{x}-1}\right)^{k}=\sum_{m=0}^{\infty} \frac{x^{m}}{m !} B_{m}^{(k)} \quad\left(B_{m}=B_{m}^{(1)}\right)
$$

For $k=p$, put

$$
A_{r}=(-1)^{r}\left(\begin{array}{c}
p-1 \\
r
\end{array}\right) B_{r}^{(p)} \quad(0 \leqq r \leqq p-1) ;
$$

then as is well known

$$
(x+1)(x+2) \cdots(x+p-1)=x^{p-1}+A_{1} x^{p-2}+\cdots+A_{p-1} .
$$

Glaisher $[4$, p. 325] has established the congruences

$$
A_{2 r} \equiv-\frac{1}{2 r} p B_{2 r}\left(\bmod p^{2}\right) \text {, }
$$

$$
A_{2 r+1} \equiv \frac{2 r+1}{4 r} p^{2} B_{2 r}\left(\bmod p^{3}\right)
$$

for $1 \leqq r \leqq(p-3) / 2, p$ prime $>3$. On the other hand the writer [1] has proved that

$$
B_{p}^{(p)} \equiv p^{2} / 2\left(\bmod p^{3}\right)
$$

and indeed [2] the more precise result

$$
B_{p}^{(p)} \equiv-p^{2}(p-1) ! / 2\left(\bmod p^{5}\right) .
$$

Now for $k=-p$, Nielsen [5, p. 338] has proved that

$$
\begin{aligned}
B_{2 r}^{(-p)} & \equiv \frac{1}{2 r} p B_{2 r}\left(\bmod p^{2}\right), \\
B_{2 r+1}^{(-p)} & \equiv \frac{2 r+1}{4 r} p^{2} B_{2 r}\left(\bmod p^{3}\right),
\end{aligned}
$$

where $1 \leqq r \leqq(p-3) / 2$.

Received by the editors June 3, 1952. 
In the next place we define the numbers $C_{m}^{(k)}$ by means of $[6$, p. 143]

$$
\left(\frac{2}{e^{x}+1}\right)^{k}=\sum_{m=0}^{\infty} \frac{C_{m}^{(k)}}{2^{m}} \frac{x^{m}}{m !} \quad\left(C_{m}=C_{m}^{(1)}\right),
$$

so that the $C_{m}^{(k)}$ are closely related to the Euler numbers of order $k$. Corresponding to (1.3) and (1.6) we have, for $k=p$,

$$
\begin{aligned}
& C_{2 r}^{(p)} \equiv p C_{2 r-1}\left(\bmod p^{2}\right), \\
& C_{2 r+1}^{(p)} \equiv-(2 r+1) p^{2} C_{2 r-1}\left(\bmod p^{3}\right),
\end{aligned}
$$

where now $r \geqq 1$. For $k=-p$, we have

$$
\begin{aligned}
& C_{2 r}^{(-p)} \equiv-p C_{2 r-1}\left(\bmod p^{2}\right), \\
& C_{2 r+1}^{(-p)}=-(2 r+1) p^{2} C_{2 r-1}\left(\bmod p^{3}\right)
\end{aligned}
$$

for $r \geqq 1$; (1.8) is due to Nielsen [5, p. 292]. We remark that (1.3), (1.6), (1.7), (1.8) are proved in a uniform manner in [3].

In view of the above it is natural to seek congruences corresponding to (1.5) for the numbers $B_{p}^{(-p)}, C_{p}^{(p)}, C_{p}^{(-p)}$. Since

$$
B_{m}^{(-k)}=\frac{m !}{(m+k) !} \sum_{r=0}^{k}(-1)^{k-r}\left(\begin{array}{l}
k \\
r
\end{array}\right) r^{m+k},
$$

it seems convenient, in analogy with (1.2), to define

$$
\bar{A}_{m}=\frac{1}{p !} \sum_{r=0}^{p}(-1)^{p-r}\left(\begin{array}{l}
p \\
r
\end{array}\right) r^{m+p}
$$

Then we shall show that

$$
\bar{A}_{p} \equiv p^{2} \bar{A}_{p-1}+\frac{1}{18} p^{5} B_{p-3}\left(\bmod p^{6}\right),
$$

while

$$
\bar{A}_{p-1} \equiv \frac{2 p B_{p-1}}{p-1}+(p-1) !\left(\bmod p^{2}\right)
$$

In particular (1.10) and (1.11) imply

$$
\bar{A}_{p} \equiv p^{2}(p-1) !+\frac{2 p^{3} B_{p-1}}{p-1}\left(\bmod p^{5}\right)
$$

In the next place for $C_{p}^{(p)}$ we prove 


$$
C_{p}^{(p)} \equiv-p^{2}(p-1) C_{p-2}\left(\bmod p^{4}\right) .
$$

For $C_{p}^{(-p)}$ we prove

$$
C_{p}^{(-p)} \equiv p^{2} C_{p-1}^{(-p)}+\frac{2}{3} p^{5} C_{p-4}\left(\bmod p^{6}\right) ;
$$

also

$$
C_{p-1}^{(-p)}+p C_{p-2} \equiv C_{p-1}^{(p)} \equiv p^{2} \sum_{1}^{p-4}\left(\begin{array}{c}
p-2 \\
s
\end{array}\right) C_{s} C_{p-3-s}\left(\bmod p^{3}\right)
$$

We suppose throughout that $p$ is a prime $>3$.

2. Proof of (1.10) and (1.11). To prove (1.10) we make use of the following formula [5, p. 293]:

$$
\bar{A}_{r}=\sum_{s=0}^{r}(-1)^{s}\left(\begin{array}{l}
p+r \\
p+s
\end{array}\right) p^{r-s} \bar{A}_{\imath}
$$

which can be proved without much trouble using (1.9). Now for $r=p,(2.1)$ becomes

$$
\begin{aligned}
2 \bar{A}_{p} & =\sum_{s=1}^{p}(-1)^{p-s}\left(\begin{array}{c}
2 p \\
s
\end{array}\right) p^{s} \bar{A}_{p-\bullet} \\
& \equiv 2 p^{2} \bar{A}_{p-1}-\left(\begin{array}{c}
2 p \\
2
\end{array}\right) p^{2} \bar{A}_{p-2}+\left(\begin{array}{c}
2 p \\
3
\end{array}\right) p^{3} \bar{A}_{p-3}\left(\bmod p^{6}\right) .
\end{aligned}
$$

But by (1.6) we have

$$
\bar{A}_{p-2} \equiv \frac{1}{3} p^{2} B_{p-3}\left(\bmod p^{3}\right), \quad \bar{A}_{p-3} \equiv-\frac{1}{3} p B_{p-3}\left(\bmod p^{2}\right),
$$

so that (2.2) yields

$$
\begin{aligned}
2 \bar{A}_{p} & \equiv 2 p^{2} \bar{A}_{p-1}+\frac{1}{3} p^{5} B_{p-3}-\frac{2}{8} p^{5} B_{p-3} \\
& \equiv 2 p^{2} \bar{A}_{p-1}+\frac{1}{9} p^{5} B_{p-3}\left(\bmod p^{6}\right)
\end{aligned}
$$

which is equivalent to (1.10).

To prove (1.11) we use the formula [6, p. 146]

$$
B_{m}^{(k)}=-\frac{k}{m} \sum_{s=1}^{m}(-1)^{\cdot}\left(\begin{array}{c}
m \\
s
\end{array}\right) B_{s} B_{m-8}^{(k)} \text {. }
$$

In (2.3) take $m=p-1, k=p$ and $-p$, and use (1.3) and (1.6); we get after a little manipulation

$$
B_{p-1}^{(-p)}-B_{p-1}^{(p)} \equiv \frac{2 p B_{p-1}}{p-1}\left(\bmod p^{3}\right)
$$


which is equivalent to (1.11).

3. Proof of (1.13). We shall require the formula $[6$, p. 146]

$$
C_{m+1}^{(k)}=-k \sum_{s=0}^{m}(-1) \cdot\left(\begin{array}{l}
m \\
s
\end{array}\right) C_{s} C_{m-8,}^{(k)}
$$

which is evidently analogous to (2.3). For $k=m+1=p$, (3.1) implies (since $C_{2 r}=0$ for $r>0$ )

$$
\begin{aligned}
\frac{1}{p} C_{p}^{(p)}= & -C_{p-1}^{(p)}+\sum_{s=1}^{p-4}\left(\begin{array}{c}
p-1 \\
s
\end{array}\right) C_{s} C_{p-1-s}^{(p)}+(p-1) C_{p-2} C_{1}^{(p)} \\
\equiv & -C_{p-1}^{(p)}-p(p-1) C_{p-2} \\
& -p^{2}(p-1) \sum_{1}^{p-4}\left(\begin{array}{c}
p-2 \\
s
\end{array}\right) C_{s} C_{p-3-s}\left(\bmod p^{8}\right)
\end{aligned}
$$

by the second of (1.7). In the next place if we take $m+1=p-1$ in (3.1), we get

$$
\begin{aligned}
C_{p-1}^{(p)} & =-p C_{p-2}^{(p)}+p \sum_{1}^{p-4}\left(\begin{array}{c}
p-2 \\
s
\end{array}\right) C_{8} C_{p-2-s}^{(p)}+p C_{p-2} C_{0}^{(p)} \\
& \equiv p^{2} \sum_{1}^{p-4}\left(\begin{array}{c}
p-2 \\
s
\end{array}\right) C_{8} C_{p-3-8}\left(\bmod p^{8}\right)
\end{aligned}
$$

by the first of (1.7). Comparison of (3.2) and (3.3) yields

$$
\begin{aligned}
\frac{1}{p} C_{p}^{(p)} & \equiv-p C_{p-1}^{(p)}-p(p-1) C_{p-2} \\
& \equiv-p(p-1) C_{p-2}\left(\bmod p^{8}\right)
\end{aligned}
$$

which is equivalent to (1.13).

4. Proof of (1.14) and (1.15). We remark first that for $k>0$ we have

$$
2^{k-m} C_{m}^{(-k)}=\sum_{s=0}^{k}\left(\begin{array}{l}
k \\
s
\end{array}\right) s^{m},
$$

by means of which it is easy to prove $[5$, p. 290]

$$
2^{k-m} C_{m}^{(-k)}=\sum_{i=0}^{m}(-1)^{\bullet}\left(\begin{array}{l}
m \\
s
\end{array}\right) 2^{k-s} k^{m-s} C_{s}^{(-k)} \text {. }
$$

In (4.1) and (4.2) we take 


$$
k=p=m
$$

then (4.2) becomes

$$
\begin{aligned}
2 C_{p}^{(-p)}= & \sum_{0=1}^{p}(-1)^{p-8}\left(\begin{array}{l}
p \\
s
\end{array}\right) 2^{8} p^{8} C_{p-8}^{(-p)} \\
\equiv & 2 p^{2} C_{p-1}^{(-p)}-2 p^{8}(p-1) C_{p-2}^{(-p)} \\
& +\frac{4}{8} p^{4}(p-1)(p-2) C_{p-3}^{(-p)} \\
\equiv & 2 p^{2} C_{p-1}^{(-p)}-2 p^{8}(p-1)(p-2) C_{p-4} \\
& -\frac{4}{8} p^{5}(p-1)(p-2) C_{p-4} \\
\equiv & 2 p^{2} C_{p-1}^{(-p)}+\frac{4}{8} p^{5} C_{p-4}\left(\bmod p^{6}\right),
\end{aligned}
$$

where we have used (1.8). This proves (1.14).

In the next place if we take $k=-p, m+1=p-1$ in (3.1) we get

$$
\begin{aligned}
C_{p-1}^{(-p)} & =p \sum_{s=0}^{p-2}(-1)^{s}\left(\begin{array}{c}
p-2 \\
s
\end{array}\right) C_{s} C_{p-2-s}^{(-p)} \\
& =-p C_{p-2}+p C_{p-2}^{(-p)}-p \sum_{1}^{p-4}\left(\begin{array}{c}
p-2 \\
s
\end{array}\right) C_{s} C_{p-2-s}^{(-p)} \\
& \equiv-p C_{p-2}+p^{2} \sum_{1}^{p-4}\left(\begin{array}{c}
p-2 \\
s
\end{array}\right) C_{s} C_{p-8-1}\left(\bmod p^{3}\right) .
\end{aligned}
$$

Comparison of (4.4) with (3.3) gives

$$
C_{p-1}^{(-p)} \equiv C_{p-1}^{(p)}-p C_{p-2}\left(\bmod p^{3}\right),
$$

which implies the first half of (1.15). The second half follows from (4.4).

5. Some of the above formulas can be simplified slightly. For example it is easy to show that

$$
\sum_{1}^{p-4}\left(\begin{array}{c}
p-2 \\
s
\end{array}\right) C_{s} C_{p-3-s}=\frac{1}{2} \sum_{1}^{p-4} C_{s} C_{p-3-s}(\bmod p) .
$$

It is not evident whether the right member of (5.1) can be reduced further. In this connection the formula

$$
C_{m+1}=\sum_{1}^{m-1}\left(\begin{array}{c}
m \\
r
\end{array}\right) C_{r} C_{m-r}
$$

may be mentioned. It follows from (5.2) that 


$$
C_{p} \equiv-\sum_{1}^{p-2} C_{p} C_{p-1 \rightarrow}(\bmod p) .
$$

In the second place we recall that $[6$, p. 28]

$$
C_{m-1}=2^{m}\left(1-2^{m}\right) \frac{B_{m}}{m} .
$$

By means of (5.3), (1.13) for example becomes

$$
C_{p}^{(p)} \equiv 2^{p-1}\left(2^{p-1}-1\right) p^{2} B_{p-1}(\bmod p)
$$

while (1.14) becomes

$$
C_{p}^{(-p)} \equiv p^{2} C_{p-1}^{(-p)}-\frac{1}{24} p^{6} B_{p-8}\left(\bmod p^{6}\right)
$$

and so on.

\section{REFERENCES}

1. L. Carlitz, Some theorems on Bernoulli numbers of higher order, Pacific Journal of Mathematics vol. 2 (1952) pp. 127-139.

2. Some congruences for Bernoulli numbers of higher order, Quarterly Journal of Mathematics.

3. - A theorem of Glaisher, Canadian Journal of Mathematics.

4. J. W. L. Glaisher, On the residues of the sums of products of the first $p-1$ numbers, and their powers, to modulus $p^{2}$ or $p^{2}$, Quarterly Journal of Mathematics vol. 31 (1900) pp. 321-353.

5. N. Nielsen, Traite elementaire des nombres de Bernoulli, Paris, 1923.

6. N. E. Nörlund, Vorlesungen uber Differenzenrechnung, Berlin, 1924.

DURE UNIVERSITY 